\title{
Ino80 promotes cervical cancer tumorigenesis by activating Nanog expression
}

\author{
Jing Hu${ }^{1}$, Jie Liu ${ }^{1}$, Aozheng Chen ${ }^{1}$, Jia Lyu ${ }^{1}$, Guihai Ai ${ }^{1}$, Qiongjing Zeng ${ }^{1}, Y_{i}$ Sun $^{1}$, \\ Chunxia Chen ${ }^{1}$, Jinbo Wang ${ }^{1}$, Jin Qiu ${ }^{1}$, Yi Wu ${ }^{1}$, Jiajing Cheng ${ }^{1,3}$, Xiujuan Shi ${ }^{2}$, \\ Liwen Song ${ }^{1}$ \\ ${ }^{1}$ Department of Obstetrics and Gynecology, Shanghai Tenth People's Hospital, Tongji University School of Medicine, Shanghai, \\ China \\ ${ }^{2}$ Shanghai Tenth People's Hospital, Tongji University School of Medicine, Shanghai, China \\ ${ }^{3}$ The First Clinical Medical College of Nanjing Medical University, Nanjing, Jiangsu, China \\ Correspondence to: Jiajing Cheng, email: chengjiajing0911@163.com \\ Xiujuan Shi, email: xiujuansh@tongji.edu.cn \\ Liwen Song, email: m18521307528@163.com
}

Keywords: Ino80, Nanog, cervical cancer, tumorigenesis, proliferation

Received: September 02, $2015 \quad$ Accepted: October 10, 2016

Published: October 14, 2016

\section{ABSTRACT}

Ino80 ATPase is an integral component of the INO80 ATP-dependent chromatinremodeling complex, which regulates transcription, DNA repair and replication. We found that Ino80 was highly expressed in cervical cancer cell lines and tumor samples. Ino80 knockdown inhibited cervical cancer cell proliferation, induced G0/G1 phase cell cycle arrest in vitro and suppressed tumor growth in vivo. However, Ino80 knockdown did not affect cell apoptosis, migration or invasion in vitro. Ino80 overexpression promoted proliferation in the $\mathbf{H} 8$ immortalized cervical epithelial cell line, which has low endogenous Ino80 expression as compared to cervical cancer cell lines. Ino80 bound to the Nanog transcription start site (TSS) and enhanced its expression in cervical cancer cells. Nanog overexpression in Ino80 knockdown cell lines promoted cell proliferation. This study demonstrated for the first time that Ino80 was upregulated in cervical cancer and promoted cell proliferation and tumorigenesis. Our findings suggest that Ino80 may be a potential therapeutic target for the treatment of cervical cancer.

\section{INTRODUCTION}

Incidences of cervical cancer in developed countries have decreased dramatically because of cytologic screening and DNA testing for high-risk human papillomavirus. However, cervical cancer remains the fourth most common cancer in females worldwide, with approximately 527,600 new cases and 265,700 deaths annually, and nearly $90 \%$ of deaths occurring in developing countries [1]. Although cures are achieved at early stages with radical surgery, chemo-radiotherapy or both, there are limited options for advanced (recurrent, persistent or metastatic) cervical cancers [2-6]. The 5-year survival rate for women with advanced disease is only $16 \%[7]$.

Aberrations in chromatin regulators, such as in histone-modifying enzymes and chromatin remodelers, are associated with diverse cancers [8-11]. Mutations in genes encoding subunits of the SWI/SNF chromatin remodeling complexes are found collectively in $20 \%$ of all human cancers, approaching the frequency of p53 mutations [9]. Genetic variations in DNA- and histone-modifying genes are novel predictive biomarkers of recurrence and survival in early stage non-small cell lung cancer patients [8].

The ATP-dependent chromatin remodeling complexes can utilize the energy of ATP hydrolysis to modulate chromatin structure, and are critically involved in processes that require DNA access such as transcription, replication and repair $[12,13]$. All of these complexes include a sucrose non-fermenting 2 (SNF2) family ATPase with ATP-dependent nucleosome remodeling activity [14]. The Ino80 ATPase is a member of the SNF2 family ATPases and is an integral component of the INO80 ATPdependent chromatin remodeling complex (INO80) [15]. 
Recent studies reveal that INO80 is involved in DNA and telomere replication, DNA repair, transcription regulation and maintenance of genome stability [16-22]. INO80 dysfunction may perturb DNA synthesis, gene regulation and DNA repair, potentially leading to genome instability and the development of cancer. A recent study demonstrates that Ino80 interacts with BRCA1associated protein-1 (BAP1), a tumor suppressor that also stabilizes Ino80, in normal DNA replication [23]. Ino80 is downregulated in BAP1-defective cancer cells due to destabilization, suggesting a molecular basis for the BAP1 tumor-suppressor function. Another study reports that Ino80 is required for efficient cell proliferation, and $I n o 80^{+/-} p 53^{-/-}$mice exhibit a striking shift from lymphomas to sarcomas compared to $p 53^{-/-}$mice [19]. This suggests that Ino80 influences tumor type.

Pluripotent transcription factors, which help maintain embryonic stem cell (ESC) self-renewal and pluripotency, are involved in tumorigenesis and progression in various cancers, including cervical cancer [24-28]. For example, Nanog knockdown by small interfering RNA (siRNA) reduces cell proliferation and induces G0/G1 cell cycle arrest in breast cancer cells [28]. High SOX2 and OCT4 expression indicates radiation resistance and poor prognosis in cervical cancer patients [27]. Oct4 directly induces miR-125b expression, inhibiting its direct target BAK1, and suppressing cervical cancer cell apoptosis [26].

Interactions between chromatin regulators and pluripotent transcription factors are essential for ESC selfrenewal and pluripotency. Tryptophan aspartic acid (WD) repeat domain 5 (WDR5), an $\mathrm{H} 3 \mathrm{~K} 4$ methylation effector, reportedly mediates self-renewal and reprogramming via binding with OCT4 in ESCs [29]. INO80 facilitates pluripotency gene activation in ESCs by binding OCT4 and WDR5 [30]. However, little is known about their interactions in cancer cells. In this study, we investigated the role of Ino80 in cervical cancer tumorigenesis, along with the related genetic and epigenetic regulatory mechanisms.

\section{RESULTS}

\section{Ino80 is highly expressed in cervical cancer}

To assess Ino80 expression in cervical cancer, we examined gene expression data from one published study consisting of 9 cervical cancer cell lines, 24 normal cervical tissues and 28 cervical cancer samples [31]. Compared to normal cervical tissues, Ino80 was markedly upregulated in cervical cancer samples (Figure 1A). Ino80 expression was higher in cervical cancers as compared to corresponding pericarcinous tissues (Figure 1B). Similarly, Ino80 expression was elevated in the human cervical cancer cell lines, HeLa, SiHa, C-33A, CaSki and
MS751, compared to the immortalized cervical epithelial cell line, H8 (Figure 1C-1D).

\section{Ino80 knockdown does not influence cervical cancer cell apoptosis}

We designed two shRNAs targeting Ino80: shIno80 A and shIno80 B. All shRNA-infected HeLa and SiHa cells expressed ZsGreen under fluorescence microscopy observation (Figure S1A-S1B). Compared with the control (scrambled shRNA), shIno80 A and B efficiently downregulated Ino80 mRNA (Figure 2A and 2E) and protein (Figure 2B and 2F) in both cell lines.

The INO80 chromatin complex is critically involved in DNA repair. Therefore, we examined whether Ino80 knockdown affected cervical cancer cell apoptosis. p53, $\mathrm{Bcl} 2$ and Bax mRNA and protein levels were unchanged following Ino80 knockdown as detected by qRT-PCR and western blot analyses (Figure 2C-2D and 2G-2H) and this was confirmed by TUNEL assay (Figure S1C-S1D). These data demonstrate that Ino80 knockdown does not impact cervical cancer cell apoptosis.

\section{Ino80 knockdown inhibits cervical cancer cell proliferation}

Ino80 was required for efficient mouse embryo fibroblast (MEF) proliferation [19]. We hypothesized that Ino80 may also promote cervical cancer cell proliferation. Ino80 knockdown inhibited HeLa and $\mathrm{SiHa}$ cell proliferation as measured via CCK-8 assay (Figure 3A and 3E). Colony formation assay showed that Ino80 knockdown in these cells decreased the number of formed colonies (Figures 3C-3D and 3G-3H). Ino80 knockdown also reduced cell viability as detected by MTT assay (Figure 3B and 3F). These data suggest that Ino80 promotes cervical cancer cell proliferation in vitro.

\section{Ino80 knockdown induces $\mathrm{G} 0 / \mathrm{G} 1$ phase cell cycle arrest}

$\mathrm{Ki} 67$ protein is a proliferation marker present during all active phases of the cell cycle (G1, S, G2 and mitosis), but absent from resting cells (G0). Ino80 knockdown in HeLa and $\mathrm{SiHa}$ cells resulted in lower Ki67 expression compared with controls (Figure 4A-4B). We used flow cytometry to determine whether Ino80 was involved in cervical cancer cell cycle regulation. Ino80 knockdown in HeLa and SiHa cells dramatically increased the cell population at $\mathrm{G} 0 / \mathrm{G} 1$ phase, and reduced the cell population at $\mathrm{S}$ and $\mathrm{G} 2 / \mathrm{M}$ phases (Figure 4C-4F). CyclinD1 expression also decreased in Ino80 knockdown cells (Figure 4G-4H). Collectively, these results demonstrate that Ino80 knockdown mainly induces G0/G1 phase cell cycle arrest in cervical cancer cells. 


\section{Ino80 knockdown does not affect cervical cancer cell migration and invasion}

Wound healing and transwell chamber migration assays suggested Ino80 knockdown in $\mathrm{HeLa}$ and $\mathrm{SiHa}$ cells did not affect cellular migration compared with controls (Figure S2A-S2B). Similarly, cell invasion through an extracellular matrix did not change following Ino80 knockdown (Figure S2B). Mmp2, Mmp9 and Mmp11 mRNA levels were also unchanged in control and Ino80 knockdown cells (Figure S2C). These data indicate that Ino80 does not regulate cervical cancer cell migration and invasion.

\section{Ino80 knockdown suppresses cervical cancer cell growth in vivo}

Stable Ino80 knockdown or control HeLa and $\mathrm{SiHa}$ cells were subcutaneously injected into female nude mice and tumor growth was measured. Growth of tumors derived from Ino80 knockdown cells was suppressed compared with controls four weeks post-inoculation (Figure 5A and 5E). Ino80 knockdown group tumor volumes and weights were reduced compared to controls (Figure 5B-5C and 5F-5G). CyclinD1 expression was also decreased in Ino80 knockdown groups (Figure 5D and $5 \mathrm{H})$. These results indicate that Ino80 promotes cervical cancer cell growth in vivo.

\section{Ino80 overexpression promotes cervical epithelial cell proliferation}

We investigated whether Ino80 knockdown inhibited proliferation in the human immortalized cervical epithelial cell line, H8. Ino80 mRNA and protein levels in $\mathrm{H} 8$ cells were decreased following Ino80 knockdown (Figure S3A and S3B). CCK8 and colony formation assays showed similar proliferation rates in control and Ino80 knockdown cells (Figure S3C-S3D). Similarly, Ki67 levels were also unchanged (Figure S3E). However, H8 cells express lower levels of endogenous Ino80 than do cervical cancer lines (Figure 1C-1D). Therefore, we overexpressed Ino80 in H8 cells (Figure 6A-6B). Ino80 overexpression promoted H8 cell proliferation as detected by CCK8 and colony formation assays (Figure 6C-6D), and enhanced Ki67 expression (Figure 6E).
A

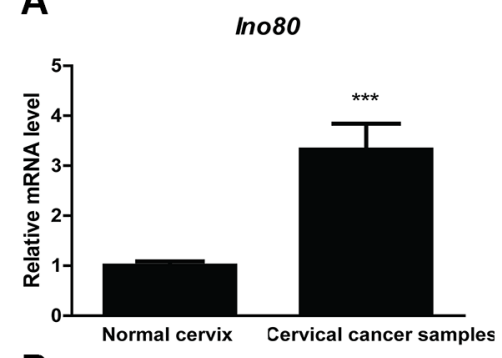

в
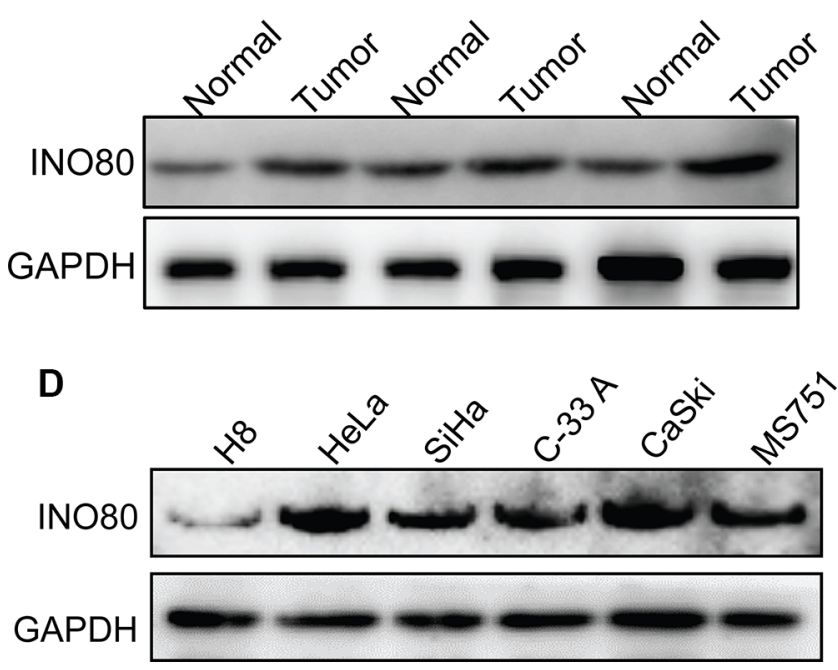

Figure 1: Ino80 is highly expressed in cervical cancer. Ino80 expression in normal cervical tissues and cervical cancer samples from one published microarray gene expression dataset (GEO Accession number: GSE9750) (A). Data are displayed relative to normal cervix group as means \pm SEM. $* * * p<0.001$. Ino80 expression in cervical cancers (tumor) and corresponding pericarcinous tissues (normal) from three patients (B). qRT-PCR analysis of Ino80 in the human immortalized cervical epithelial cell (H8) and cervical cancer cell lines (HeLa, SiHa, C-33A, CaSki and MS751) (C). Data are displayed relative to H8 as means \pm SEM $(n=3) .{ }^{*} p<0.05, * * p<0.01$, $* * * p<0.001$. Western blot analysis of Ino80 in H8, HeLa, SiHa, C-33A, CaSki and MS751 cells with GAPDH as a loading control (D). 


\section{Ino80 promotes Nanog expression by binding its transcription start site}

Ino80 bound to pluripotency gene promoter proximal regions of Oct4 and Nanog to activate their expression, and Ino80 knockdown decreased expression of these key pluripotency factors in ESCs [30]. Oct4 and Nanog reportedly play pivotal roles in cervical cancer progression [26, 32, 33]. We hypothesized that Ino80 may promote cervical cancer tumorigenesis through elevating expression of these pluripotency factors. We found that Oct4 expression was very low in HeLa cells (data not showed), consistent with a previous study [34]. We measured Ino80 binding to Oct4 and Nanog gene transcription start sites (TSS) and upstream sites via ChIP assay followed by quantitative PCR (ChIP-qPCR) and found that Ino80 was present at Nanog, but not Oct4 TSS (Figure 7A). Consistent with our observations in cervical cancer cells, Nanog knockdown by siRNA reportedly reduced proliferation and induced G0/G1 cell cycle arrest in breast cancer cells [28]. Compared with controls, we found that Ino80 knockdown in HeLa cells reduced Nanog expression (Figure 7B-7C) and decreased Ino80 binding in the Nanog TSS (Figure 7D).
A

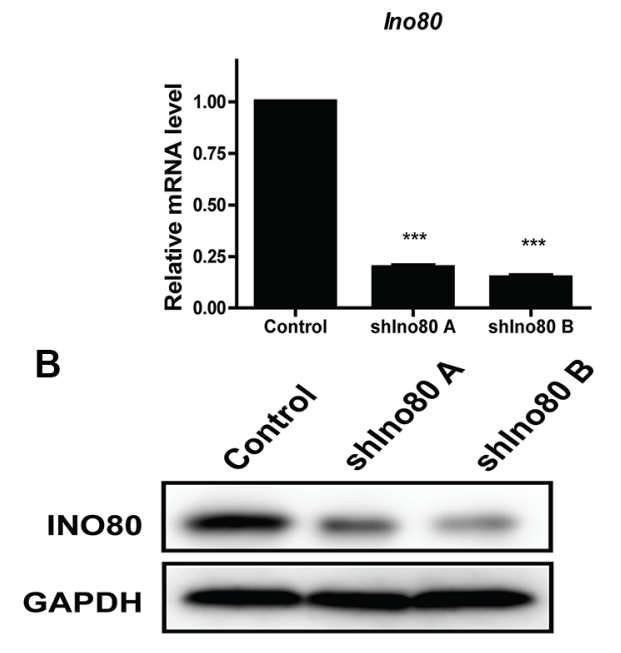

C

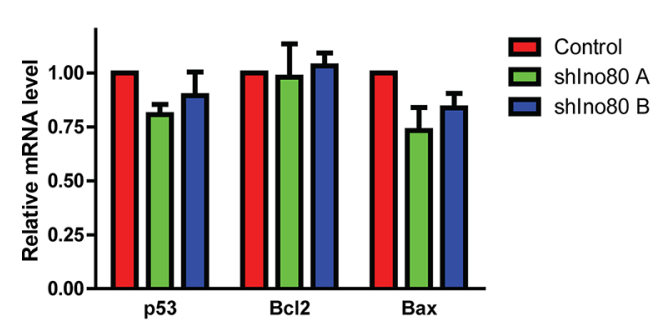

D

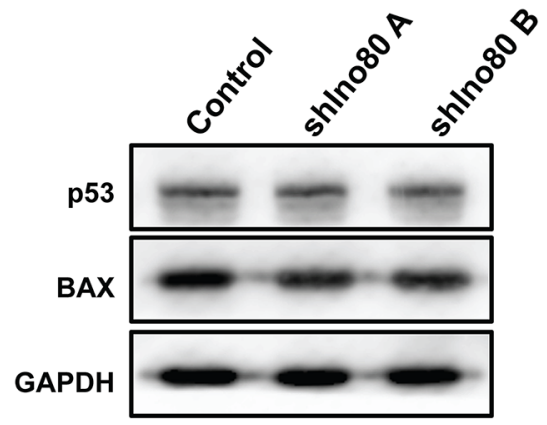

E

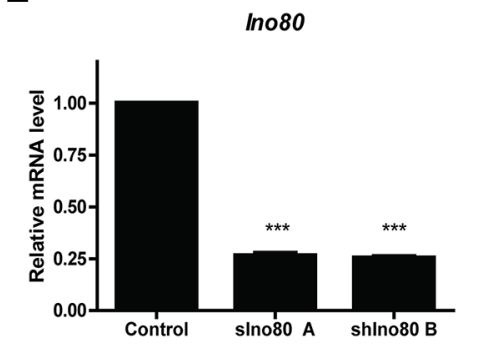

$\mathbf{F}$

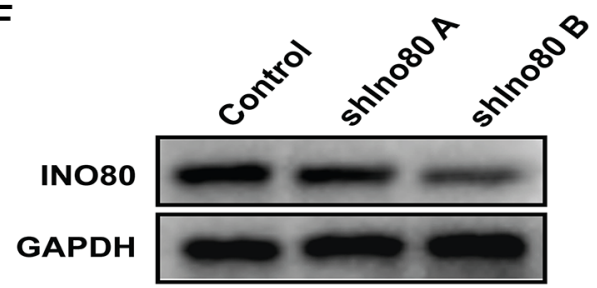

G

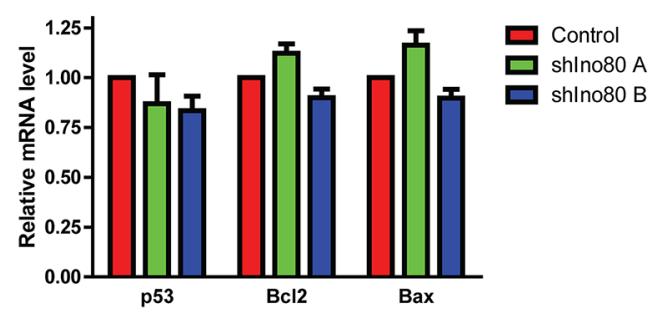

H

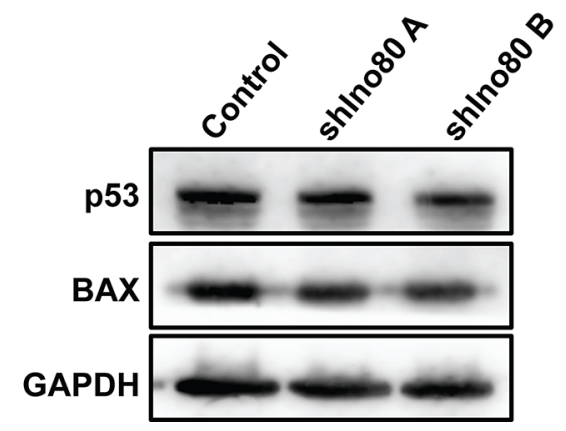

Figure 2: Ino80 knockdown and cell apoptosis detection. qRT-PCR analysis of Ino80 in control (scrambled shRNA) and Ino80 knockdown HeLa (A) and SiHa (E) cells. Western blot analysis of Ino80 in control and Ino80 knockdown HeLa (B) and SiHa (F) cells. qRT-PCR analysis of p53, Bcl2 and Bax in control and Ino80 knockdown HeLa (C) and SiHa (G) cells. Western blot analysis of p53 and BAX in control and Ino80 knockdown HeLa $(\mathbf{D})$ and $\mathrm{SiHa}(\mathbf{H})$ cells. Western blots used GAPDH as a loading control. qRT-PCR data are displayed relative to controls as means $\pm \operatorname{SEM}(n=3) . * * * p 0.001$. 


\section{Nanog overexpression promotes cervical cancer cell proliferation}

We overexpressed Nanog in Ino80 knockdown HeLa cells (Figure 8A-8B). CCK8 and colony formation assays showed that Ino80 knockdown decreased HeLa cell growth rates (Figure 8C-8D). However, Nanog overexpression in Ino80 knockdown HeLa cells promoted cell growth to levels comparable to control (no knockdown) cells (Figure 8C-8D), and elevated Ki67 expression (Figure 8E).

Collectively, these findings indicate that Ino80 binds to the Nanog TSS and enhances its expression in cervical cancer cells to promote tumorigenesis.

\section{DISCUSSION}

Our data demonstrated that Ino80 promoted cervical cancer cell proliferation in vitro and tumor growth in vivo. Min, et al. reported that Ino80 was required for efficient MEF proliferation, and Ino80 deletion reduced proliferation [19]. Wang, et al. also reported that Ino80 was required for ESC self-renewal [30]. Our observations are consistent with these reports, and confirm a role for Ino80 in cervical cancer cell proliferation.

Stemness factor Nanog has been identified as a tumorigenic factor and is associated with poor prognosis in many cancer types [35-39]. Nanog was highly expressed
A

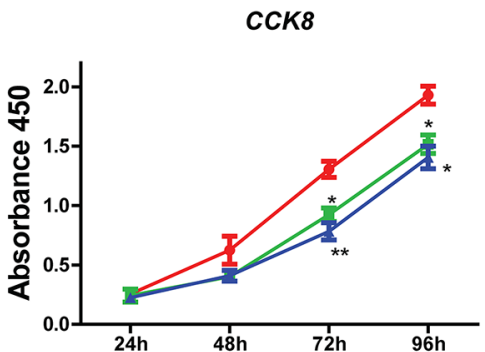

B

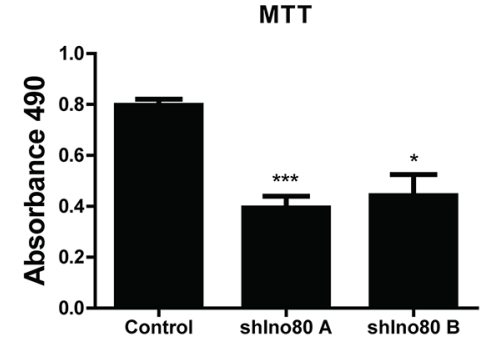

C

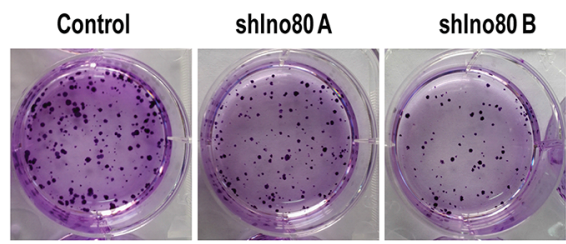

D

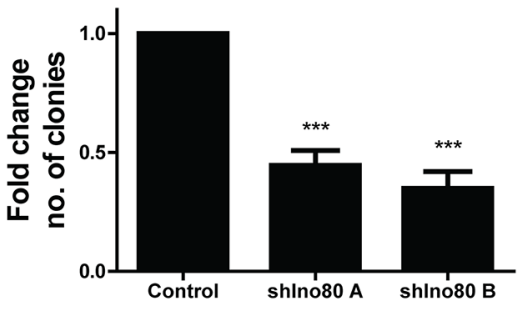

E

$F$
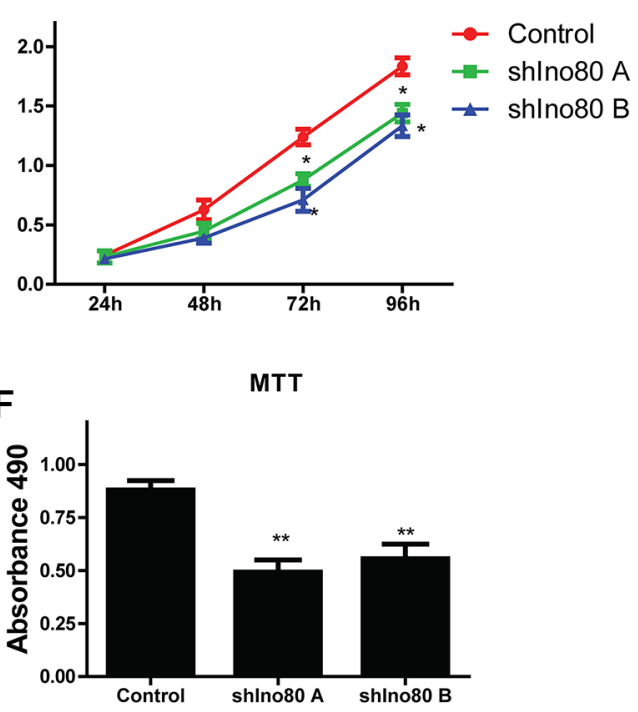

$\mathbf{G}$

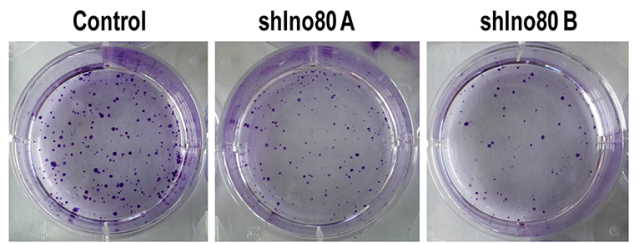

H

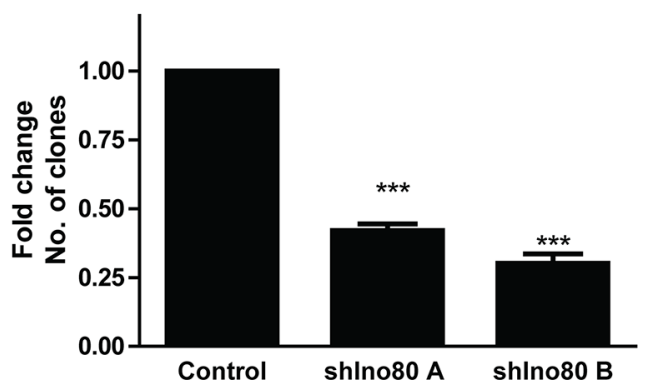

Figure 3: Ino80 knockdown inhibits cell proliferation. Growth curves of control and Ino80 knockdown HeLa (A) and SiHa (E) cells constructed from CCK-8 assay results. Influence of Ino80 knockdown on HeLa (B) and SiHa (F) cell viability as measured by MTT assay $48 \mathrm{~h}$ post-cell seeding. The data in CCK-8 and MTT assays are represented as means $\pm \operatorname{SEM}(n=3) .{ }^{*} p<0.05, * * p<0.01$, $* * * p<0.001$. Representative images of colonies formed by control and Ino80 knockdown HeLa (C) and SiHa (G) cells, stained with crystal violet. Fold change in number of colonies formed by HeLa (D) and $\mathrm{SiHa}(\mathbf{H})$ cells. Data are represented relative to controls as means \pm $\operatorname{SEM}(n=3) . * * * p<0.001$. 
in patients with squamous cervical carcinomas [40]. Our data demonstrated that Ino80 bound Nanog transcription start site and activated its expression in cervical cancer cells. We also showed that Nanog overexpression in Ino80 knockdown cervical cancer cells promoted cell proliferation. Our data suggests that Nanog may mediate the pro-proliferative effects of Ino80 in cervical cancer cell.

In this study, we demonstrated for the first time that Ino80 was upregulated in cervical cancer and promoted tumorigenesis. Our findings suggest that Ino80 may be a potential therapeutic target for cervical cancer.

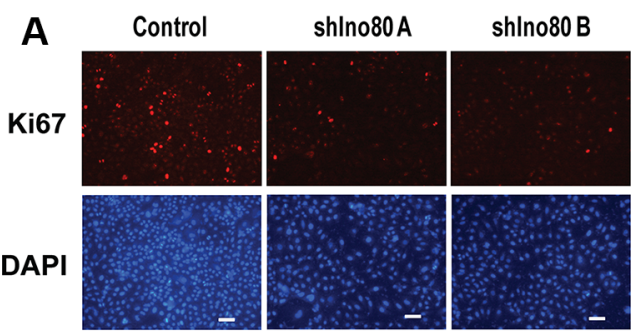

C
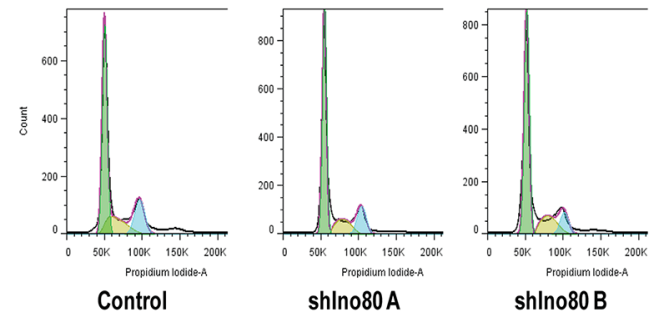

$\mathbf{E}$
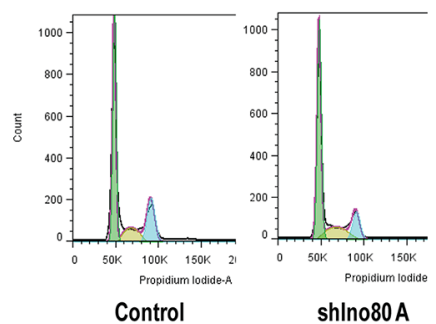

G
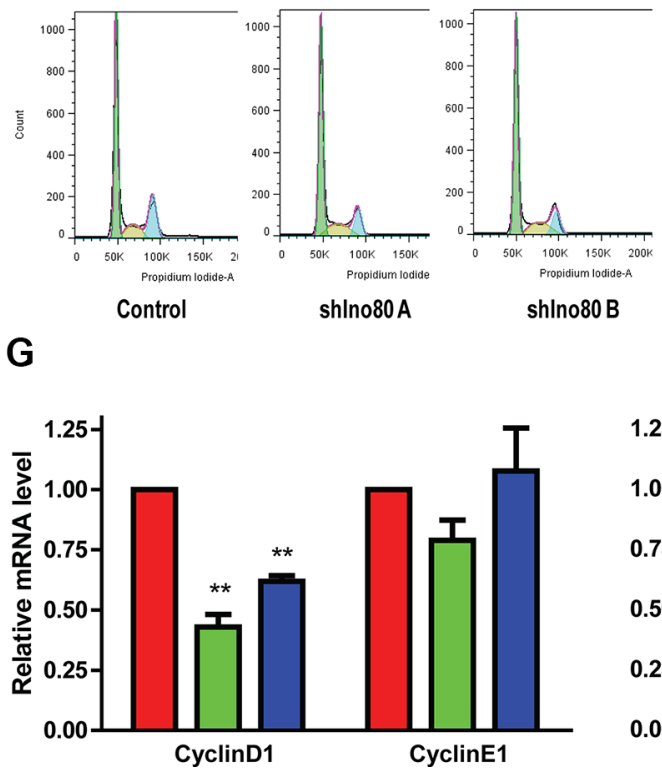

\section{MATERIALS AND METHODS}

\section{Cell culture}

Human cervical cancer cell lines (HeLa, SiHa, C-33A, CaSki and MS751) and a human immortalized cervical epithelial cell line (H8) were cultured in highglucose Dulbecco's modified Eagle's medium (DMEM) (Gibco, Grand Island, NY) supplemented with 10\% fetal bovine serum (FBS) (Gibco) and 1\% penicillin/ streptomycin (Gibco). Cells were grown in a humidified atmosphere of $5 \% \mathrm{CO} 2$ at $37^{\circ} \mathrm{C}$.

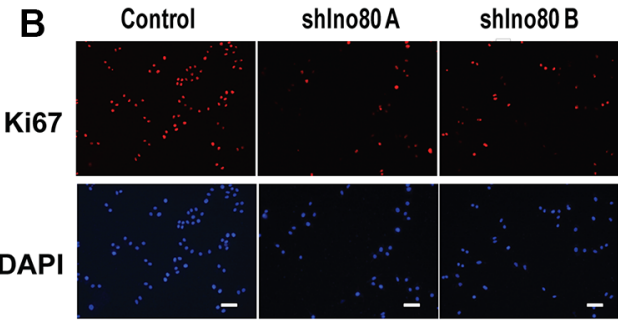

D

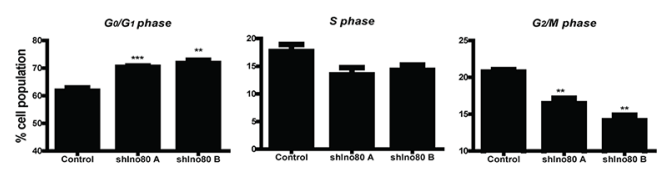

$\mathbf{F}$

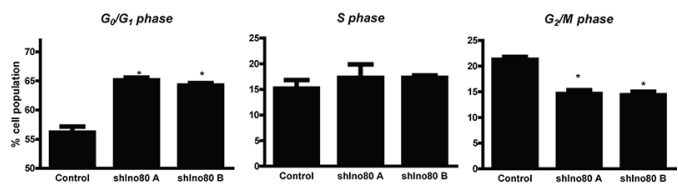

H

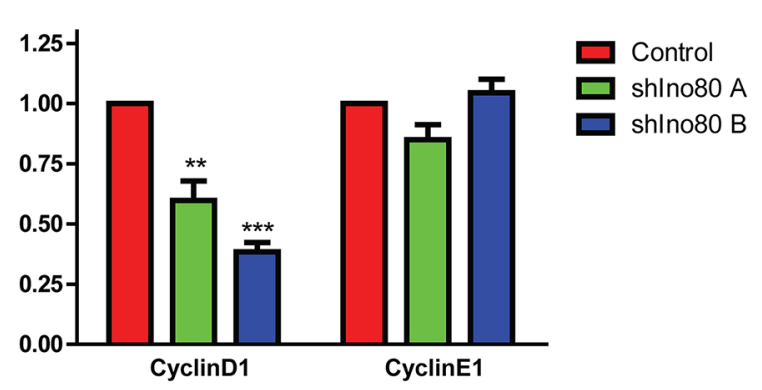

Figure 4: Ino80 knockdown induces G0/G1 phase cell cycle arrest. Ki67 expression in control and Ino80 knockdown HeLa (A) and $\mathrm{SiHa}$ (B) cells as determined by IF staining. Nuclei were counterstained with DAPI. Bars $=40 \mu \mathrm{m}$. Effect of In80 knockdown on cell cycle progression in $\mathrm{HeLa}(\mathbf{C})$ and $\mathrm{SiHa}(\mathbf{E})$ cells. FACS data were analyzed by ModFit LT software. Histograms show the percentage $(\%)$ of HeLa (D) and SiHa (F) cell populations at different cell cycle stages. Data are represented as means \pm SEM $(n=3) . * p<0.05$, ${ }^{* *} p<0.01,{ }^{* * *} p<0.001$. qRT-PCR analysis of cyclinD1 and cyclinE1 in control and Ino80 knockdown HeLa $(\mathbf{G})$ and $\mathrm{SiHa}(\mathbf{H})$ cells. Data are represented relative to controls as means $\pm \operatorname{SEM}(n=3) .{ }^{* *} p<0.01,{ }^{* * *} p<0.001$. 


\section{Patients and specimens}

Three pairs of surgically resected cervical cancer and corresponding pericarcinous tissues were obtained from patients without preoperative treatment at Tenth People's Hospital, Tongji University School of Medicine (Shanghai, China) between September 2014 and September 2015. Human specimen collection procedures were approved by the Ethics Committee of Tenth People's Hospital.

\section{Plasmids and lentivirus constructs}

Ino80 shRNA knockdown (shIno80) sequences were obtained from Sigma-Aldrich (Table S1). shIno80 sequences were ligated into the PLVX-shRNA2 vector expressing a classic scrambled shRNA and green fluorescent protein (ZsGreen). Ino80 and Nanog overexpression sequences (Table S2) were ligated into PLVX-IRES-TDTOMATO and FUGW-H1-GFP vectors, respectively. For viral packaging, 293T cells were cotransfected with lentiviral plasmids using the FuGene HD transfection reagent (Roche Diagnostics, Basel, Switzerland). Virus-containing medium was harvested 48 or $72 \mathrm{~h}$ post-transfection, then filtered to remove cell debris and used for infection. To generate knockdown or overexpression cell lines, $1 \times 10^{5}$ cells were seeded into six-well plates $1 \mathrm{~d}$ before infection. Medium was then replaced with virus-containing supernatant supplemented with $8 \mu \mathrm{g} / \mathrm{ml}$ polybrene (Sigma, St. Louis, MO). Ino80
A

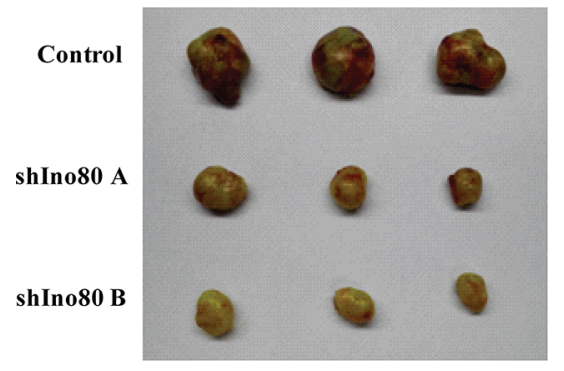

B

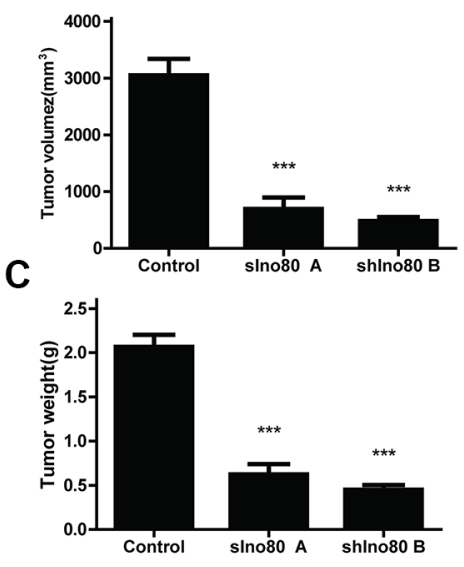

D

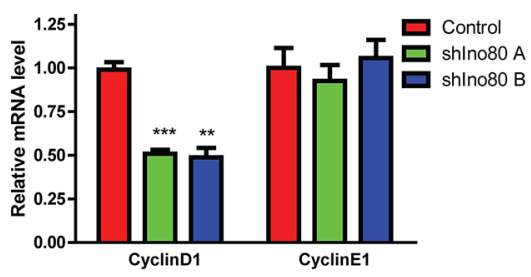

E

$\mathbf{F}$

G

H
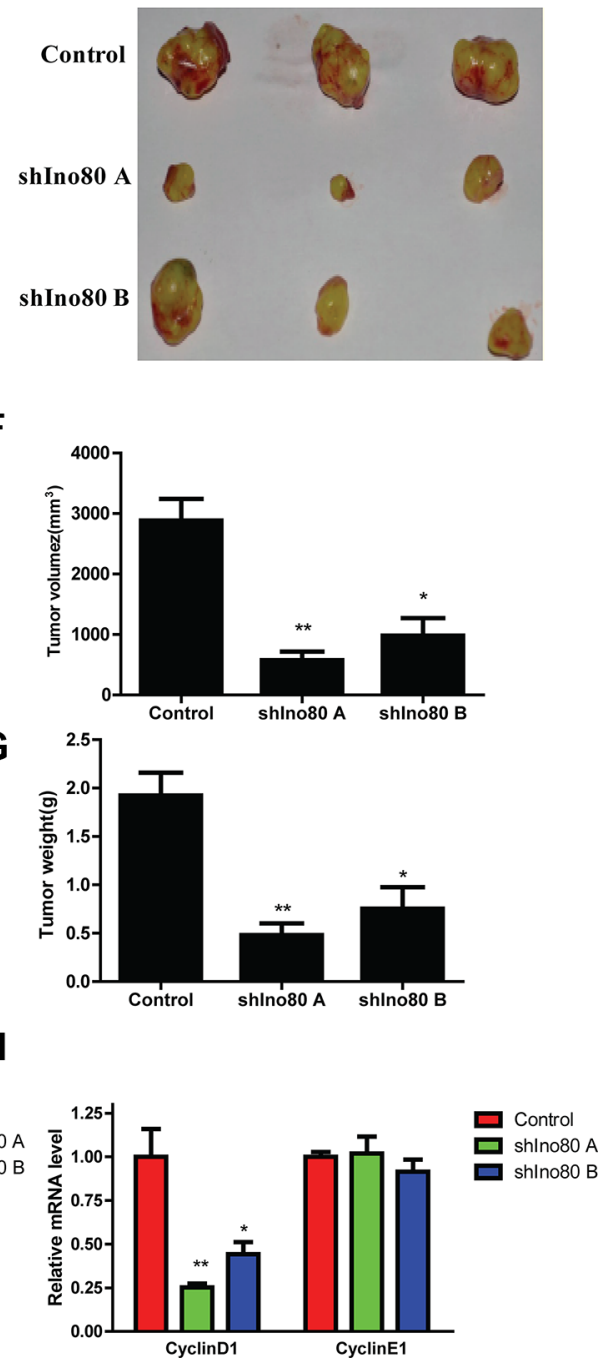

Figure 5: Ino80 knockdown suppresses cervical cancer cell growth in vivo. Images of tumors derived from control and Ino80 knockdown $\mathrm{HeLa}(\mathbf{A})$ and $\mathrm{SiHa}(\mathbf{E})$ cells four weeks post-inoculation. Control and Ino80 knockdown HeLa $(\mathbf{B})$ and $\mathrm{SiHa}(\mathbf{F})$ cell tumor volumes four weeks post-inoculation. Control and Ino80 knockdown $\mathrm{HeLa}(\mathbf{C})$ and $\mathrm{SiHa}(\mathbf{G})$ cell tumor weights four weeks postinoculation. Data are represented as means $\pm \operatorname{SEM}(n=3) .{ }^{*} p<0.05,{ }^{* *} p<0.01,{ }^{* * *} p<0.001$. qRT-PCR analysis of cyclinD1 and cyclinE1 in control and Ino80 knockdown HeLa (D) and SiHa (H) cell groups. Data are represented relative to controls as means $\pm \operatorname{SEM}(n=3)$. ${ }^{*} p<0.05,{ }^{* *} p<0.01,{ }^{* * *} p<0.001$. 
knockdown and overexpression cells were selected through Flow cytometry using a FACSCalibur system (BD Biosciences). Nanog overexpression cells were selected with G418 (Sigma, St. Louis, MO).

\section{Cell proliferation assay}

Methyl thiazolyl tetrazolium (MTT) assay was used to screen for cell viability. Cells were seeded in 96-well plates at $2 \times 10^{3}$ cells/well. $48 \mathrm{~h}$ later, absorbance was measured at a wavelength of $490 \mathrm{~nm}$ using a SpectraMax M5 plate reader (Molecular Devices).

For the Cell Counting Kit-8 (CCK8) cell proliferation assay (Dojindo, Kumamoto, Japan), $1 \times 10^{3}$ cells/well were seeded in 96-well plates. After 24, 48, 72 and $96 \mathrm{~h}$, the CCK8 reagent was thawed for approximately $10 \mathrm{~min}$ in a water bath at $37^{\circ} \mathrm{C}$, and $10 \mu \mathrm{l}$ of the reagent was added to each well. Plates were incubated at $37^{\circ} \mathrm{C}$ for $1-4 \mathrm{~h}$. The absorbance at $450 \mathrm{~nm}$ was recorded using a SpectraMax M5 plate reader.
For colony formation assay, 500 cells/well were seeded in a 6-well plate. Approximately $14 \mathrm{~d}$ later, clones were fixed in $100 \%$ methanol and stained with $1 \%$ crystal violet in $\mathrm{ddH}_{2} \mathrm{O}$. Clones were then imaged and quantified.

For cell cycle analysis, cells were fixed in $70 \%$ ice cold ethanol followed by RNase A treatment. Cells were stained with $50 \mu \mathrm{g} / \mathrm{ml}$ propidium iodide for DNA content analysis in a BD FACSCalibur flow cytometer. Data were collected using BD FACSuite analysis software and analyzed using ModFit LT software (Verity Software House, Inc).

\section{Wound healing assay}

Culture inserts (ibidi, Martinsried, Germany) consisting of two reservoirs separated by a 500-mm thick wall were placed in a 24 -well plate. An equal amount $(70 \mu \mathrm{l})$ of cell suspension $\left(5 \times 10^{5}\right.$ cells $\left./ \mathrm{ml}\right)$ was added to each reservoir followed by incubation at $37^{\circ} \mathrm{C}$. After cell attachment $(10 \mathrm{~h})$, culture inserts were gently removed

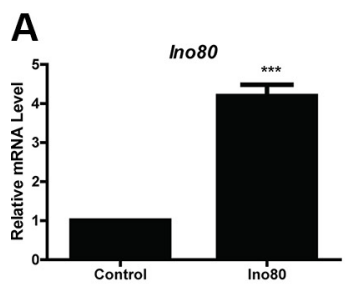

C

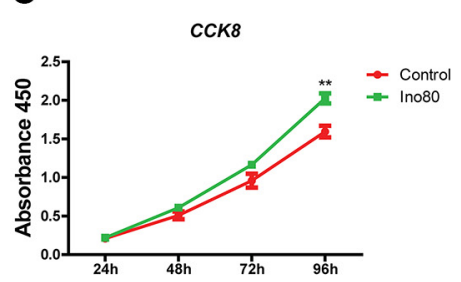

E

Control

B

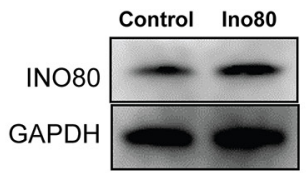

D

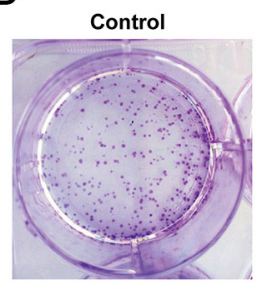

Ino80
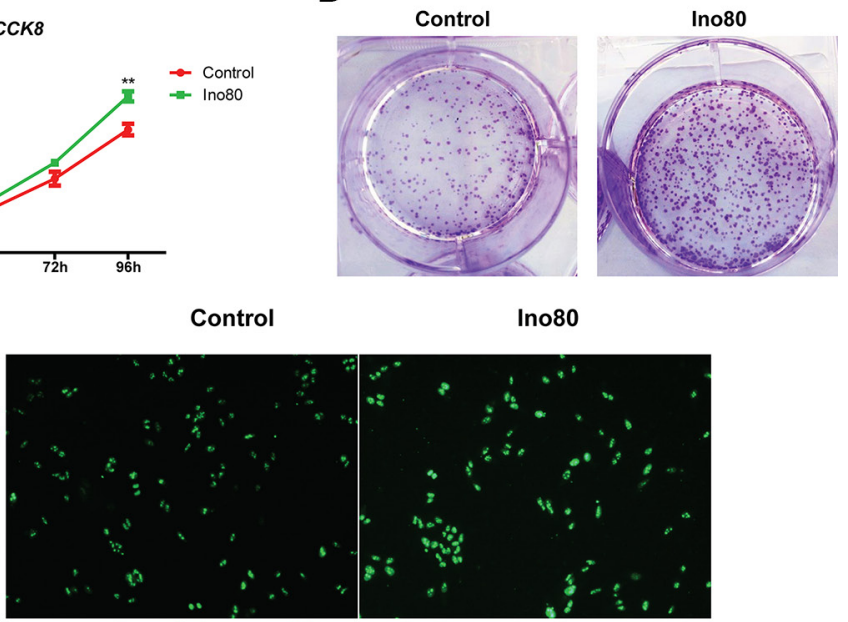

DAPI

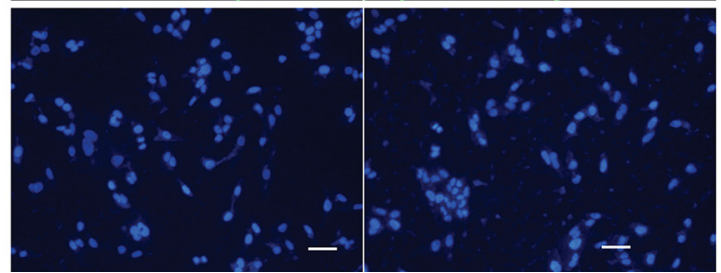

Figure 6: Ino80 overexpression promotes cervical epithelial cell proliferation. qRT-PCR (A) and western blot (B) analyses of Ino80 in control and Ino80 overexpression H8 cells. qRT-PCR data are represented relative to control as mean \pm SEM $(n=3)$. $*_{* *} p<0.001$. Western blot analysis used GAPDH as a loading control. Control and Ino80 overexpression H8 cell growth curves constructed using CCK-8 assay results $(\mathbf{C})$ Data are represented as means $\pm \operatorname{SEM}(n=3)$. $* * p<0.01$. Colony formation in control and Ino80 overexpression $\mathrm{H} 8$ cells (D) Colonies were stained with crystal violet. Ki67 expression in control and Ino80 overexpression H8 cells as determined by IF staining (E) Nuclei were counterstained with DAPI. Bars $=40 \mu \mathrm{m}$. 
and wells were filled with serum-free culture medium containing $0.2 \%$ bovine serum albumin (BSA). The gap between two cell layers was observed under an inverted microscope immediately and after $36 \mathrm{~h}$.

\section{Transwell assay}

Cell migration and invasion were analyzed using transwell chambers (Corning, NY, USA). For migration, HeLa and SiHa cells were suspended in DMEM with 1\% FBS and added to the upper chambers $\left(1 \times 10^{5}\right.$ cells/well $)$, which were incubated at $37^{\circ} \mathrm{C}$ for $24 \mathrm{~h}$. Cells on the upper surface of the membrane were then removed. Membranes were fixed with $4 \%$ paraformaldehyde and cells on the undersurface were stained with DAPI. The chambers were observed under a fluorescence microscope. For invasion, chambers were pre-coated with matrigel (BD Biosciences; $50 \mathrm{mg} / \mathrm{ml} ; 1: 8)$ at $37^{\circ} \mathrm{C}$ for $4 \mathrm{~h}$. After cells were added, chambers were incubated at $37^{\circ} \mathrm{C}$ for $24 \mathrm{~h}$.

\section{TUNEL assay}

Terminal deoxynucleotidyl transferase dUTP nick end labeling (TUNEL) assay was performed using the onestep TUNEL apoptosis assay kit from Beyotime Institute of Biotechnology in China. Cells treated as indicated were fixed with 4\% paraformaldehyde and permeabilized with $0.25 \%$ Triton X-100. Cells were incubated with $50 \mu 1$ TUNEL reaction mixture for $1 \mathrm{~h}$ at $37^{\circ} \mathrm{C}$ in the dark. Cells were observed under a fluorescent microscope (550 $\mathrm{nm}$ excitation and $570 \mathrm{~nm}$ emission) and images were captured and digitized by image analysis software.

A
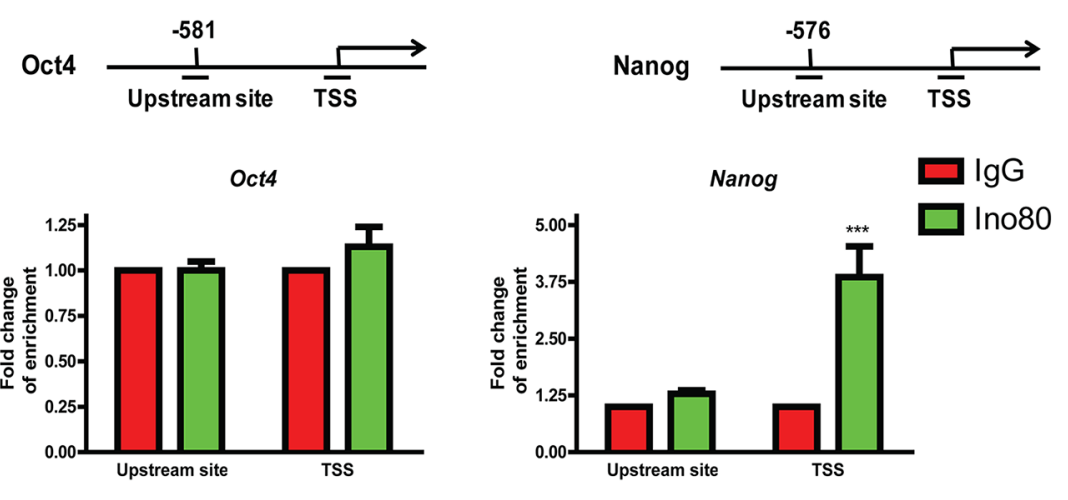

B
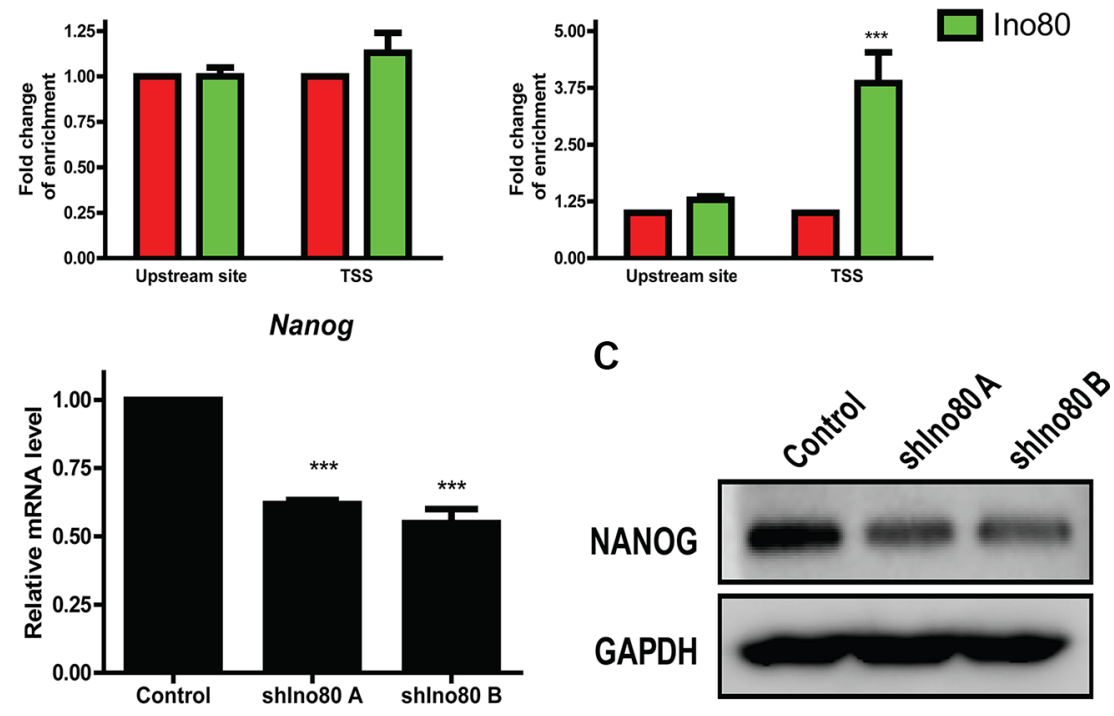

D

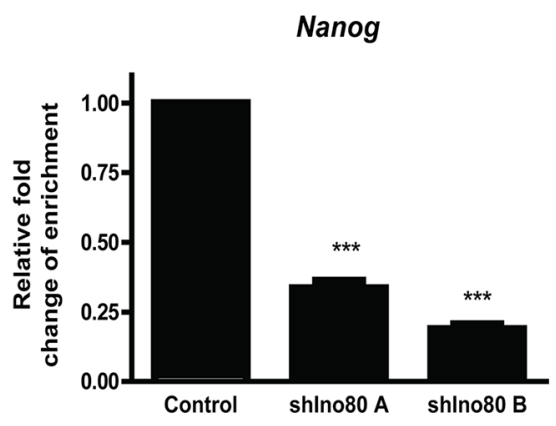

Figure 7: Ino80 promotes Nanog expression by binding to its transcription start site (TSS). ChIP-qPCR analysis showing Ino80 binding levels in the Oct4 and Nanog gene TSS and upstream sites in HeLa cells (A) Enrichment fold changes are normalized to IgG binding and is represented as mean $\pm \operatorname{SEM}(n=3)$. ${ }^{* * *} p<0.001$. qRT-PCR $(\mathbf{B})$ and western blotting $(\mathbf{C})$ analysis of Nanog in control and Ino80 knockdown HeLa cells. Western blot analysis used GAPDH as a loading control. ChIP-qPCR analysis showing Ino80 binding levels in the Nanog TSS in control and Ino80 knockdown HeLa cells (D) Data in B and D are represented relative to controls as means \pm SEM $(n=3) . * * * p<0.001$. 


\section{Immunofluorescence (IF) staining}

Cells cultured in 24-well plates were fixed in $4 \%$ paraformaldehyde and permeabilized with $0.25 \%$ Triton $\mathrm{X}-100$, followed by blocking with $10 \%$ FBS in PBS. Cells were then probed with primary anti-Ki67 antibody (Abcam, Cambridge, UK) in $10 \% \mathrm{FBS}$ overnight at $4^{\circ} \mathrm{C}$, then secondary antibody in $10 \%$ FBS for $2 \mathrm{~h}$ at room temperature. Cells were counterstained with DAPI for $2 \mathrm{~min}$. Cells were washed with and imaged in PBS.

A

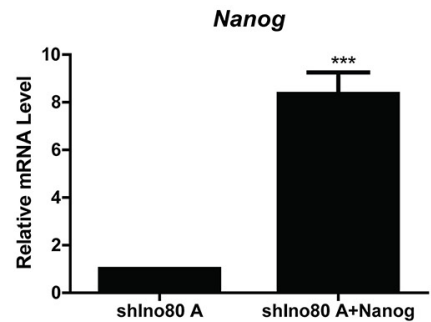

C

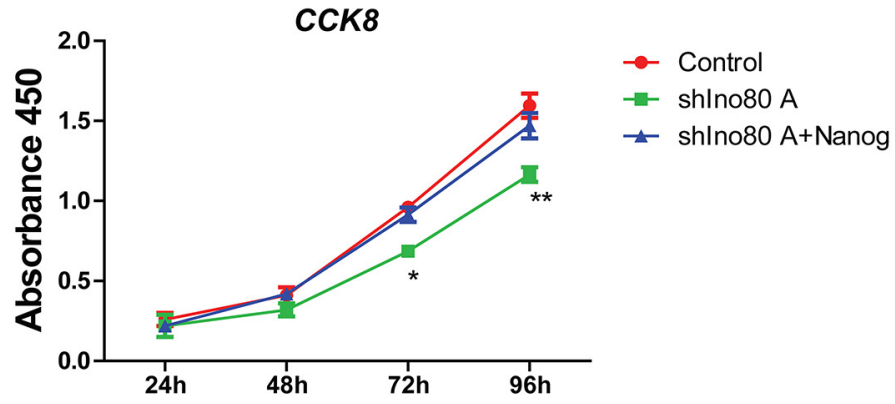

D

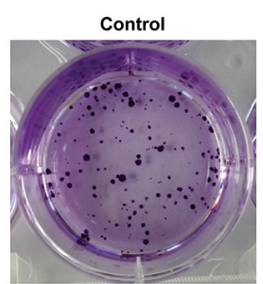

$\mathbf{E}$

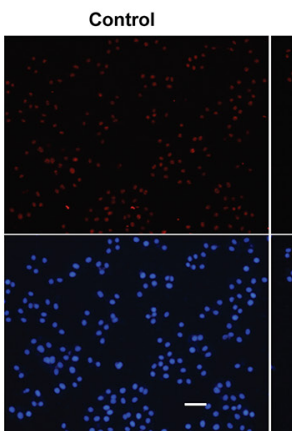

\section{Western blotting}

Cultured cells were lysed in strong RIPA buffer containing Halt Protease Inhibitor Cocktails (Thermo, Waltham, MA). Protein concentrations were measured using a BCA protein assay kit (Pierce, Rockford, IL). Primary antibodies targeting NANOG (Abcam), Ino80 (proteintech, Chicago, IL), OCT4 (Santa Cruz Biotechnology, Santa Cruz, CA), p53 (Santa Cruz Biotechnology), BAX (Abcam) and GAPDH (Santa Cruz Biotechnology) were

B
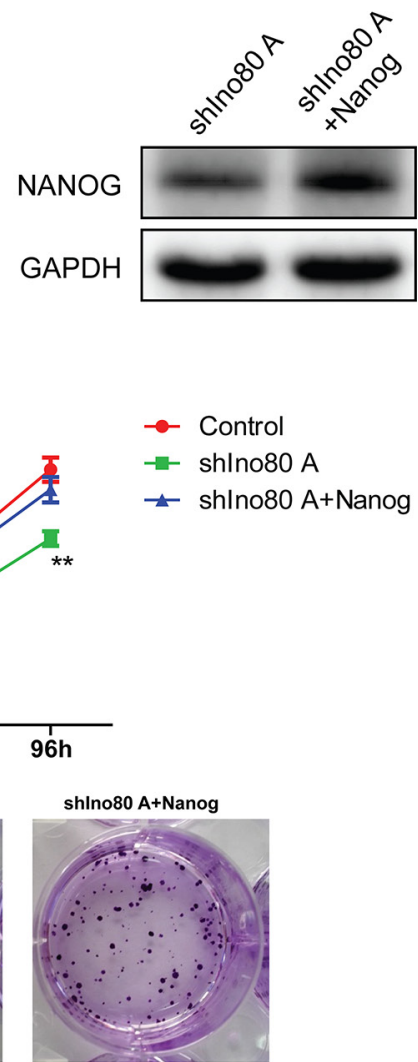

shlno80 A

shlno80 A+Nanog

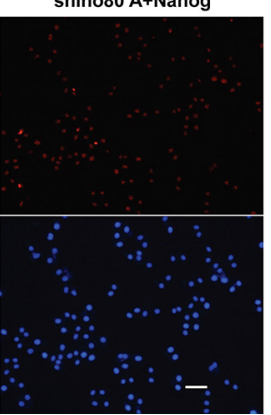

Figure 8: Nanog overexpression promotes cervical cancer cell proliferation. Cells are divided into three groups: HeLa cell (control), Ino80 knockdown HeLa cell (shIno80 A) and Nanog overexpression in Ino80 knockdown HeLa cell (shIno80 A + Nanog). qRTPCR (A) and western blotting (B) analysis of Nanog in the shIno80 A and shIno80 A + Nanog groups. qRT-PCR data are displayed relative to the shIno80 A group as mean $\pm \operatorname{SEM}(n=3)$. $* * * p<0.001$. Western blot analysis used GAPDH as a loading control. Control, shIno80 $\mathrm{A}$ and shIno80 A + Nanog HeLa cell growth curves constructed from CCK-8 assay results (C). Data are represented as means \pm SEM $(n=3) .{ }^{*} p<0.05, * * p<0.01$. Colony formation in control, shIno80 A and shIno80 A + Nanog HeLa cells (D). Colonies were stained with crystal violet. Ki67 expression in control, shIno80 A and shIno80 A + Nanog HeLa cells as determined by IF staining (E) Nuclei were counterstained with DAPI. Bars $=40 \mu \mathrm{m}$. 
incubated with the proteins overnight at $4^{\circ} \mathrm{C}$, followed by incubation with the appropriate HRP (horseradish peroxidase)-conjugated secondary antibodies. Detection of HRP was performed using the Super Signal West Pico Chemiluminescent Substrate (Pierce).

\section{Reverse transcription and $q$ RT-PCR}

Total RNA was isolated using the Trizol reagent (Invitrogen, Carlsbad, CA) following the manufacturer's instructions. For each sample, $500 \mathrm{ng}$ of RNA was reverse transcribed to cDNA using the Prime-Script RT reagent kit (TaKaRa, Dalian, China). cDNA was amplified with the Takara Ex Taq PCR kit (TaKaRa). qRT-PCR amplification was conducted using the Stratagene Mx3000 QPCR system (Stratagene, Foster City, CA) and analyzed via the $\Delta \Delta \mathrm{CT}$ method. Primer sequences are provided in Table S3.

\section{Chromatin immunoprecipitation assay}

Chromatin immunoprecipitation (ChIP) assays were performed with normal rabbit IgG (Millipore, Billerica, MA) and Ino80 (Proteintech) antibody using the EZ ChIP kit (Millipore) according to the manufacturer's protocol. Whole-cell DNA and immunoprecipitated DNA were used for PCR assays with primers targeting sequences surrounding the binding sites. Primer sequences are provided in Table S4. Fold enrichment was calculated relative to normal rabbit $\mathrm{IgG}$.

\section{Animal studies}

All in vivo experiments were performed according to approved protocols from the Institutional Animal Care and Use Committee of Tongji University. $5 \times 10^{6} \mathrm{HeLa}$ and $\mathrm{SiHa}$ cells were injected subcutaneously into the right or left forelimb axillaries of three 4-week-old female nude mice. All mice were killed after four weeks and subcutaneous tumor nodules were extracted and measured.

\section{Statistical analysis}

Error bars represent the SEM of three independent experiments. Data are represented as means $\pm \mathrm{SEM} ; n=3$. $p<0.05$ was considered statistically significant (Student's $t$ test).

\section{CONFLICTS OF INTEREST}

The authors declare no conflicts of interest.

\section{GRANT SUPPORT}

This work was supported by the National Natural Science Foundation of China (Grant No.81502451), the Shanghai Committee of Science and Technology, China
(Grant No. 15ZR1432600 and Grant No. 15695841100) and the Fundamental Research Funds for the Central Universities. We are grateful to Bo Zhang for his foundation financial support (the National Natural Science Foundation of China, Grant No.81202045). The funders had no role in study design, data collection and analysis, decision to publish, or preparation of the manuscript.

\section{REFERENCES}

1. Torre LA, Bray F, Siegel RL, Ferlay J, Lortet-Tieulent J, Jemal A. Global cancer statistics, 2012. CA Cancer J Clin. 2015; 65:87-108.

2. Monk BJ, Sill MW, McMeekin DS, Cohn DE, Ramondetta LM, Boardman CH, Benda J, Cella D. Phase III trial of four cisplatin-containing doublet combinations in stage IVB, recurrent, or persistent cervical carcinoma: a Gynecologic Oncology Group study. J Clin Oncol. 2009; 27:4649-4655.

3. Tewari KS, Monk BJ. The rationale for the use of nonplatinum chemotherapy doublets for metastatic and recurrent cervical carcinoma. Clin Adv Hematol Oncol. 2010; 8:108-115.

4. Tewari KS, Monk BJ. Recent achievements and future developments in advanced and recurrent cervical cancer: trials of the Gynecologic Oncology Group. Semin Oncol. 2009; 36:170-180.

5. Long HJ, 3rd, Bundy BN, Grendys EC, Jr., Benda JA, McMeekin DS, Sorosky J, Miller DS, Eaton LA, Fiorica JV, Gynecologic Oncology Group S. Randomized phase III trial of cisplatin with or without topotecan in carcinoma of the uterine cervix: a Gynecologic Oncology Group Study. J Clin Oncol. 2005; 23:4626-4633.

6. Moore DH, Tian C, Monk BJ, Long HJ, Omura GA, Bloss JD. Prognostic factors for response to cisplatinbased chemotherapy in advanced cervical carcinoma: a Gynecologic Oncology Group Study. Gynecol Oncol. 2010; 116:44-49.

7. Siegel RL, Miller KD, Jemal A. Cancer statistics. CA Cancer J Clin. 2015; 65:5-29.

8. Wagner KW, Ye Y, Lin J, Vaporciyan AA, Roth JA, Wu X. Genetic variations in epigenetic genes are predictors of recurrence in stage I or II non-small cell lung cancer patients. Clin Cancer Res. 2012; 18:585-592.

9. Helming KC, Wang X, Roberts CW. Vulnerabilities of mutant SWI/SNF complexes in cancer. Cancer cell. 2014; 26:309-317.

10. Mar BG, Bullinger L, Basu E, Schlis K, Silverman LB, Dohner K, Armstrong SA. Sequencing histone-modifying enzymes identifies UTX mutations in acute lymphoblastic leukemia. Leukemia. 2012; 26:1881-1883.

11. Wang X, Haswell JR, Roberts CW. Molecular pathways: SWI/SNF (BAF) complexes are frequently mutated in 
cancer-mechanisms and potential therapeutic insights. Clin Cancer Res. 2014; 20:21-27.

12. Clapier CR, Cairns BR. The biology of chromatin remodeling complexes. Annu Rev Biochem. 2009; 78:273-304.

13. Hargreaves DC, Crabtree GR. ATP-dependent chromatin remodeling: genetics, genomics and mechanisms. Cell Res. 2011; 21:396-420.

14. Flaus A, Martin DM, Barton GJ, Owen-Hughes T. Identification of multiple distinct Snf2 subfamilies with conserved structural motifs. Nucleic Acids Res. 2006; 34:2887-2905.

15. Chen L, Conaway RC, Conaway JW. Multiple modes of regulation of the human Ino80 SNF2 ATPase by subunits of the INO80 chromatin-remodeling complex. Proc Natl Acad Sci USA. 2013; 110:20497-20502.

16. Park EJ, Hur SK, Kwon J. Human INO80 chromatinremodelling complex contributes to DNA double-strand break repair via the expression of Rad54B, XRCC3 genes. Biochem J. 2010; 431:179-187.

17. Hur SK, Park EJ, Han JE, Kim YA, Kim JD, Kang D, Kwon J. Roles of human INO80 chromatin remodeling enzyme in DNA replication and chromosome segregation suppress genome instability. Cell Mol Life Sci. 2010; 67:2283-2296.

18. Gospodinov A, Vaissiere T, Krastev DB, Legube G, Anachkova B, Herceg Z. Mammalian Ino80 mediates double-strand break repair through its role in DNA end strand resection. Mol Cell Biol. 2011; 31:4735-4745.

19. Min JN, Tian Y, Xiao Y, Wu L, Li L, Chang S. The mINO80 chromatin remodeling complex is required for efficient telomere replication and maintenance of genome stability. Cell Res. 2013; 23:1396-1413.

20. Conaway RC, Conaway JW. The INO80 chromatin remodeling complex in transcription, replication and repair. Trends Biochem Sci. 2009; 34:71-77.

21. Morrison AJ, Shen X. Chromatin remodelling beyond transcription: the INO80 and SWR1 complexes. Nat Rev Mol Cell Biol. 2009; 10:373-384.

22. Watanabe S, Peterson CL. The INO80 family of chromatinremodeling enzymes: regulators of histone variant dynamics. Cold Spring Harb Symp Quant Biol. 2010; 75:35-42.

23. Lee HS, Lee SA, Hur SK, Seo JW, Kwon J. Stabilization and targeting of INO80 to replication forks by BAP1 during normal DNA synthesis. Nat Commun. 2014; 5:5128.

24. Kawamura N, Nimura K, Nagano H, Yamaguchi S, Nonomura N, Kaneda Y. CRISPR/Cas9-mediated gene knockout of NANOG, NANOGP8 decreases the malignant potential of prostate cancer cells. Oncotarget. 2015; 6:22361-74. doi: 10.18632/oncotarget.4293.

25. Yin X, Li YW, Jin JJ, Zhou Y, Ren ZG, Qiu SJ, Zhang BH. The clinical and prognostic implications of pluripotent stem cell gene expression in hepatocellular carcinoma. Oncol Lett. 2013; 5:1155-1162.
26. Wang YD, Cai N, Wu XL, Cao HZ, Xie LL, Zheng PS. OCT4 promotes tumorigenesis and inhibits apoptosis of cervical cancer cells by miR-125b/BAK1 pathway. Cell Death Dis. 2013; 4:e760.

27. Shen L, Huang X, Xie X, Su J, Yuan J, Chen X. High Expression of SOX2 and OCT4 Indicates Radiation Resistance and an Independent Negative Prognosis in Cervical Squamous Cell Carcinoma. J Histochem Cytochem. 2014; 62:499-509.

28. Han J, Zhang F, Yu M, Zhao P, Ji W, Zhang H, Wu B, Wang Y, Niu R. RNA interference-mediated silencing of NANOG reduces cell proliferation and induces G0/G1 cell cycle arrest in breast cancer cells. Cancer Lett. 2012; 321:80-88.

29. Ang YS, Tsai SY, Lee DF, Monk J, Su J, Ratnakumar K, Ding J, Ge Y, Darr H, Chang B, Wang J, Rendl M, Bernstein E, et al. Wdr5 mediates self-renewal and reprogramming via the embryonic stem cell core transcriptional network. Cell. 2011; 145:183-197.

30. Wang L, Du Y, Ward JM, Shimbo T, Lackford B, Zheng X, Miao YL, Zhou B, Han L, Fargo DC, Jothi R, Williams CJ, Wade PA, et al. INO80 facilitates pluripotency gene activation in embryonic stem cell self-renewal, reprogramming, and blastocyst development. Cell stem cell. 2014; 14:575-591.

31. Scotto L, Narayan G, Nandula SV, Arias-Pulido H, Subramaniyam S, Schneider A, Kaufmann AM, Wright JD, Pothuri B, Mansukhani M, Murty VV. Identification of copy number gain and overexpressed genes on chromosome arm $20 q$ by an integrative genomic approach in cervical cancer: potential role in progression. Genes Chromosomes Cancer. 2008; 47:755-765.

32. Noh KH, Lee YH, Jeon JH, Kang TH, Mao CP, Wu TC, Kim TW. Cancer vaccination drives Nanog-dependent evolution of tumor cells toward an immune-resistant and stem-like phenotype. Cancer Res. 2012; 72:1717-1727.

33. Noh KH, Kim BW, Song KH, Cho H, Lee YH, Kim JH, Chung JY, Kim JH, Hewitt SM, Seong SY, Mao CP, Wu TC, Kim TW. Nanog signaling in cancer promotes stem-like phenotype and immune evasion. J Clin Invest. 2012; 122:4077-4093.

34. Cantz T, Key G, Bleidissel M, Gentile L, Han DW, Brenne A, Scholer HR. Absence of OCT4 expression in somatic tumor cell lines. Stem cells. 2008; 26:692-697.

35. Wang D, Lu P, Zhang H, Luo M, Zhang X, Wei X, Gao J, Zhao Z, Liu C. Oct-4 and Nanog promote the epithelialmesenchymal transition of breast cancer stem cells and are associated with poor prognosis in breast cancer patients. Oncotarget. 2014; 5:10803-10815. doi: 10.18632/ oncotarget. 2506.

36. Chang TS, Wu YC, Chi CC, Su WC, Chang PJ, Lee KF, Tung TH, Wang J, Liu JJ, Tung SY, Kuo LM, Ho HN, Ling TY, et al. Activation of IL6/IGFIR confers poor prognosis of $\mathrm{HBV}$-related hepatocellular carcinoma through induction of OCT4/NANOG expression. Clin Cancer Res. 2015; 21:201-210. 
37. Kim J, Liu Y, Qiu M, Xu Y. Pluripotency factor Nanog is tumorigenic by deregulating DNA damage response in somatic cells. Oncogene. 2016; 35:1334-40.

38. Lu X, Mazur SJ, Lin T, Appella E, Xu Y. The pluripotency factor nanog promotes breast cancer tumorigenesis and metastasis. Oncogene. 2014; 33:2655-2664.

39. Palla AR, Piazzolla D, Alcazar N, Canamero M, Grana O, Gomez-Lopez G, Dominguez O, Duenas M, Paramio JM,
Serrano M. The pluripotency factor NANOG promotes the formation of squamous cell carcinomas. Sci Rep. 2015; $5: 10205$.

40. Ye F, Zhou C, Cheng Q, Shen J, Chen H. Stem-cellabundant proteins Nanog, Nucleostemin and Musashi1 are highly expressed in malignant cervical epithelial cells. BMC cancer. 2008; 8:108. 